\title{
JThE28
}

\section{Optical Biopsy of Human Skin with Backward-Collected Optical Harmonics Signals}

\author{
Shih-Peng Tai, Tsung-Han Tsai \\ Graduate Institute of Electro-Optical Engineering, National Taiwan University, Taipei, TAIWAN Phone :886-2-23659703, FAX :886-2- \\ 23677467,E-mail : d92941004@ntu.edu.tw \\ Wen-Jeng Lee \\ Department of Electrical Engineering, National Taiwan University and Department of Medical Imaging, National Taiwan University Hospital
} Taipei, TAIWAN

Hsin-Yi Huang
Department of Pathology, National Taiwan University Hospital, Taipei, TAIWAN

Yi-Hua Liao

Department of Dermatology, National Taiwan University Hospital, Taipei, TAIWAN

Kenneth Yi-Jie Zhan,and Hsiang-Lin Liu

Graduate Institute of Physics and Department of Physics, National Taiwan Normal University, Taipei, TAIWAN

Chi-Kuang Sun

Graduate Institute of Electro-Optical Engineering and Department of Electrical Engineering, National Taiwan University, Taipei, TAIWAN

\begin{abstract}
Combining backward-collected second- and third-harmonic signals, noninvasive optical biopsy of human skin is demonstrated. With backward collection geometry and energyconservation characteristic of harmonic generations, this technique is ideal for future noninvasive clinical skin disease diagnosis.

(C) 2005 Optical Society of America

OCIS codes: (170.1870) Dermatology; (180.6900) Three-dimensional microscopy; (180.5810)

Scanning microscopy; (190.4160) Multi-harmonic generation
\end{abstract}

Traditional biopsy requires the removal, fixation, and staining of tissues from the human body. Its procedure is invasive and painful. Noninvasive in vivo optical biopsy is thus desired, which should provide highly penetrative three-dimensional (3D) sub-surface images with a sub-micron spatial resolution. Optical biopsy based on scanning two-photon fluorescence microscopy (TPFM) [1,2] has previously been demonstrated for noninvasive biopsy of skin with sub-micron lateral resolution, low out-of-focus damage, and intrinsic three-dimensional (3D) section capability. Previous two-photon fluorescence biopsy of skin $[1,2]$ based on a $780 \mathrm{~nm}$ femtosecond light provides high resolution imaging from the skin surface through the epidermal-dermal junction. However for future clinical applications without surgery, current 700-850 nm based laser scanning technology still presents several limitations including low penetration depth, in-focus cell damages, multi-photon phototoxicity due to high optical intensity in the $800 \mathrm{~nm}$ wavelength region, and toxicity if exogenous fluorescence markers were required.

Here we demonstrate an optical biopsy of human skin based on backward-collected second- and third-harmonic signals using a femtosecond Cr:forsterite laser centered at $1230 \mathrm{~nm}$. According to previous studies, light attenuation (including both absorption and scattering) in human skin [3] reaches a minimum around $1200-1300 \mathrm{~nm}$ due to the combination of diminishing scattering cross-section with wavelength and avoiding the resonant molecular absorption of common tissue constituents such as water, melanin, and hemoglobin. Besides, moving the excitation wavelength longer than $1200 \mathrm{~nm}$ makes second harmonic generation (SHG) and third harmonic generation (THG) signals detectable due to visible SHG and THG wavelength that can avoid strong UV region absorption in humantissues. Higher harmonics generation is known to leave no energy deposition to the interacted matters due to their energy-conservation characteristic. This energy-conservation characteristic provides the "noninvasive" nature desirable for clinical imaging. Due to its nonlinear nature, the generated SHG and THG intensities depend on square and cubic of the incident light intensity. Similar to multi-photon induced fluorescence process, this nonlinear dependency allows localized excitation and is ideal for intrinsic optical sectioning in optical biopsy applications [4]. 


\section{JThE28}

The demonstration of the backward-collection type harmonics optical biopsy (B-HOB) of human skin was performed using a modelocked Cr:forsterite laser centered at $1230 \mathrm{~nm}$ with a $140 \mathrm{fs}$ pulsewidth at a $110 \mathrm{MHz}$ repetition rate. The thickness of the fixed human skin sample was $\sim 1 \mathrm{~mm}$. Figure 1 shows an example of the vertically sectioned $(\mathrm{x}-\mathrm{z}) \mathrm{B}-\mathrm{HOB}$ images taken from the human epidermis and dermis based on backward collected multi-harmonic generation signals. From the vertically sectioned B-HOB images, the general histological structures in both epidermis and dermis layers can be identified through the THG modality due to its sensitivity to local optical inhomogeneity. For example, the coverglass-formalin interface, the stratum corneum, and the basal layer can all be easily picked up through the THG modality (for example, see Fig.1 (b)). SHG, on the other hand, mainly reflects the distribution of connected tissues in the dermis layer (bottom of Fig. 1(a)) due to strong backward-SHG generation in collagen fibers. Through the SHG image (for example, see Fig.1 (a)), other structures like the dermal papillary surrounded by connected tissues can also be clearly identified.

Figure 2 shows horizontally sectioned (x-y) B-HOB image (THG modality) taken from epidermis. In this figure, micro-structures through all epidermis can be clearly resolved through THG with a sub-micron resolution. Figure 3 shows another horizontally sectioned B-HOB image ( $\mathrm{SHG}$ modality) taken from the dermis layer at a depth of 150 $\mu \mathrm{m}$ from the skin surface. While SHG displays the rich collagen fibril structures in the dermal layer, other histological structures like dermal papillary can also be identified. More HOB images in human skin showing different histological structures, including blood cells and cancer cells, will be presented in this talk.

In summary, current $800 \mathrm{~nm}$ based two-photon optical biopsy technology still presents several limitations including finite penetration depth, in-focus cell damages, and phototoxicity due to the required high optical intensity and multi-photon absorption processes. Harmonics generation, however, can provide a truly non-invasive biopsy tool with excellent three-dimensional spatial resolution due to their virtual transition and nonlinear characteristics. Combining with a 1200-1300-nm light source, our study suggests that this technique could provide sub-micron resolution deep-tissue non-invasive biopsy images in human skins without using fluorescence and exogenous markers. Due to backward propagating detection geometry, this technique is ideal for non-invasive clinical biopsy of human skin diseases and even useful for the early diagnosis of skin cancer symptom such as the angiogenesis.

This work is sponsored by National Health Research Institute of Taiwan through NHRI-EX93-9201EI.

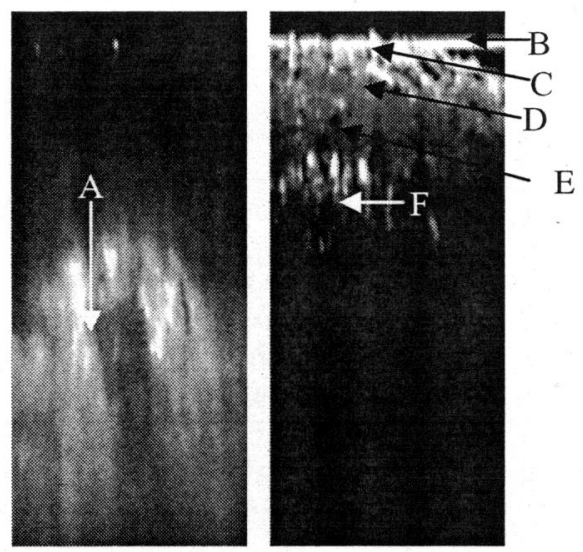

Fig.1 Vertically sectioned B-HOB images taken from the human epidermis and dermis corresponding to emitted (a) backward SHG and (b) backward THG. Image size: 120x273 $\mu \mathrm{m}$. In the figures, A-F are dermal papillary, coverglass-formalin interface, stratum corneum, stratum granulosum, stratum spinosum, and basal layer. 


\section{JThE28}
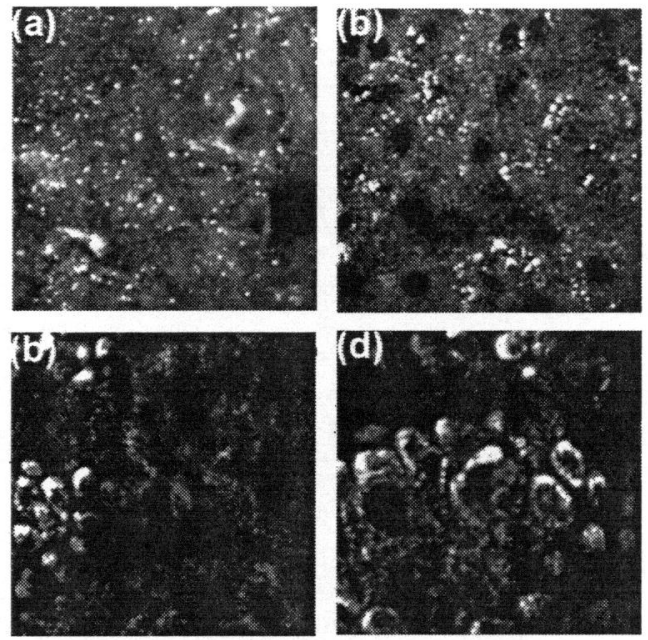

Fig.2 Horizontally sectioned B-HOB images taken from (a) stratum corneum, (b) stratum granulosum, (c) stratum spinosum, and (d) basal layer of a fixed human skin sample, based on the backward-THG modality. Image size: $80 \times 80 \mu \mathrm{m}$

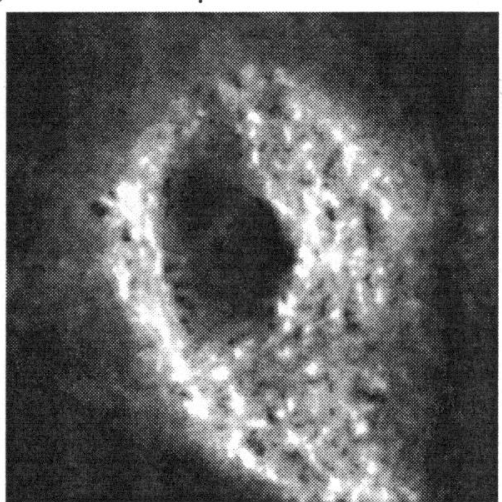

Fig. 3 Horizontally sectioned B-HOB image taken from the human dermis based on the backwardSHG modality. Image size: $120 \times 120 \mu \mathrm{m}$.

\section{References}

[1] P. T. C. So, H. Kim, and I. E. Kochevar, "Two photon deep tissue ex vivo imaging of mouse dermal and subcutaneous structures," Optics Express 3, 339 (1998).

[2] B. R. Masters and P. T. C. So, "Confocal microscopy and multi-photon excitation microscopy of human skin in vivo," Optics Express 8, 2 (2001).

[3] R. R. Anderson and J. A. Parish, "The optics of human skin," Journal of Investigative Dermatology 77, 13 (1981)

[4] C.-K. Sun, C.-C. Chen, S.-W. Chu, T.-H. Tsai, Y.-C. Chen, and B.-L. Lin, "Multiharmonic generation biopsy of skin," Optics Letters 28, 2488 (2003) 